PALAVRAS. Revista de Epistemología, Metodología y Ética del Psicoanálisis ISSN: 2468-9831

Licencia de Cultura Libre Creative Commons (C-BY 4.0).

https://revistas.unlp.edu.ar/palavras

palavras@outlook.com.ar

\title{
ESCUCHAR, PENSAR, ENSEÑAR
}

https://doi.org/10.24215/24689831e036

Luce Irigaray

\section{Cómo citar este artículo:}

Irigaray, L. (2020). Escuchar, pensar, enseñar (Trad. L, Szeinfeld). Palauras. Revista de Epistemología, Metodología y Ética del Psicoanálisis, (6), e036. https://doi.org/10.24215/24689831e036 


\section{ESCUCHAR, PENSAR, ENSEÑAR}

\section{Autor: Luce Irigaray}

Traducción: Luciana Szeinfeld ${ }^{1 *}$

Título original: Listening, thinking, teaching. Publicado en: L., Irigaray y M., Green (eds.), (2008), Teaching. London: Continuum. ${ }^{2}$

\section{Escuchar}

La tradición occidental se basa en mirar en lugar de escuchar. En nuestra tradición, escuchar está al servicio de mirar, especialmente en lo que respecta a la enseñanza. A partir de un momento determinado de la cultura griega, el maestro ha enseñado a sus discípulos lo que él ya ha percibido; es decir, lo que ya ha visto. El mundo entero se transforma, de alguna manera, en un conjunto de objetos que el maestro tiene que percibir -es decir, verde un modo apropiado, y ordenar dentro de un mundo paralelo gracias a su lenguaje, su lógica lingüística, su logos. Las discusiones, los presuntos diálogos, entre maestro y discípulo, están basados en la correcta percepción de las cosas u objetos del mundo y su correcta distribución en un todo. Gracias a su logos, el maestro construye una especie de duplicación, una suerte de duplicación mental, de la realidad externa. Él toma el mundo externo y construye una especie de mundo paralelo que siempre mantiene a mano.

Ciertamente, la construcción de este mundo siempre a mano es algo extremadamente dificil: la pregunta es cómo transformar una realidad

\footnotetext{
${ }^{1}$ Centro Interdisciplinario de Investigaciones en Género (CInIG- FaHCE- UNLP). Argentina. Mail: lucianaszeinfeld@gmail.com

2 Conferencia de Luce Irigaray en la Universidad de Nottingham (Junio 2006). Publicado en: L., Irigaray y M., Green (eds.) (2008). Teaching. London: Continuum.
} 
presente, múltiple y variada, en un único mundo siempre a mano. Esto requiere el establecimiento de una escala de valores vinculados entre sí, en la cual el valor más permanente suplantará a la realidad más presente, viva e impermanente. Por lo tanto, las discusiones o los presuntos diálogos entre el maestro y el discípulo se desarrollan en base a la relevancia de la designación o denominación de alguna cosa del mundo y a su integración en la escala de valores, pero ambos se mantienen fieles a un modo de relacionarse en el cual los dos están sujetos a un tercer mundo que se les impone y que es considerado como la verdad que ellos deben adoptar para ingresar en un mundo cultural.

Mayormente nuestra forma de enseñar permanece cercana a la de Sócrates, con la diferencia de que Sócrates estaba construyendo una nueva cultura mientras que nosotros estamos obedeciendo a una tradición que ya existe. El/la maestro/a transmite así al discípulo ese mundo paralelo construido que él, o ella, ha heredado de su propio maestro, y que convirtió en su propio mundo. Este mundo es considerado como la real y única verdad porque se corresponde con el mundo a mano adoptado por el maestro en nuestra tradición.

Actualmente este modo de enseñar ya no es apropiado. Presupone que sólo un mundo puede alcanzar la verdad universal, y no tiene en cuenta que existen diferentes mundos los cuales no conciben la verdad de la misma manera. No hay duda, por ejemplo, que Sócrates y Buda no comparten la misma concepción de la verdad, y que sería dificil decidir cuál es la mejor verdad a fin de introducir sus perspectivas en una única escala. Sus verdades son diferentes y tenemos que respetar esas diferencias si queremos respetar la verdad en sí misma.

En nuestra era, se vuelve obvio que la verdad no es ni única ni universal, y que debemos tener en cuenta varios puntos de vista y construcciones del mundo. ¿Cómo podemos afrontar esta situación? En parte al sustituir escuchar por mirar en cualquier diálogo, por lo menos invirtiendo el privilegio de uno con respecto a otro. Por supuesto, la cuestión entonces 
consiste en no simplemente escuchar a una verdad abstracta y presuntamente universal que deberiamos compartir luego de nuestra discusión, una verdad que podriamos transformar en imágenes mentales universales, sino en escuchar la manera en la cual el otro concibe y construye su verdad.

Poco a poco, esto deshace nuestra escala de valores y elabora otra relación con la verdad en la cual los dos mundos se entrelazan. El problema para nosotros, en tanto occidentales, es que ésta ya no es una cuestión de maestría, pero una en la cual nosotros ahora debemos permitir que sea hecha así como hacerla, dejar ser así como ser. Entramos entonces imperceptiblemente en otra lógica, en otra cultura.

Sin duda, la cuestión es compleja. Por ejemplo, escuchar a alguien de otra cultura nos obliga a cuestionar el lenguaje utilizado por cada uno. Pasar de un lenguaje a otro es el gesto más importante para entrar en el multiculturalismo. Esto también representa la resistencia o dificultad más importante que debemos enfrentar cuando coexistimos y compartimos culturas. Esta resistencia y dificultad yace en nuestra relación con el lenguaje. El nacionalismo más dificil de superar es el nacionalismo lingüístico. Estamos moldeados por el lenguaje: no es sólo una cuestión de entender cada palabra. Las palabras están dotadas de distintos significados en cada lenguaje: más concreto o abstracto, más sensato o mental, más subjetivo u objetivo, más relacional o solipsista, más en relación con un objeto o con otro sujeto. Cuando busco la traducción de una palabra francesa en el diccionario de inglés, frecuentemente me sorprendo por la diferencia en el significado atribuido por anglófonos a un término similar. Entonces, la cuestión es no solamente escuchar palabras, sino además el contexto lingüístico y cultural en el que éstas tienen lugar, el mundo que ellas componen y construyen.

Pasar de un lenguaje a otro requiere de una escucha que no es sólo escuchar las palabras, que supuestamente transmiten un significado universal. El asunto es que uno acepte ser cuestionado por un significado 
diferente, por un mundo cuyo sentido permanece invisible a nosotros pero que acordamos recibir, por el que aceptamos ser cuestionados y afectados al escucharlo. Escuchar, entonces, no significa tomar algo a fin de integrarlo y ordenarlo al interior de nuestro propio mundo, sino abrir nuestro propio mundo a algo o alguien externo y extraño al mismo. Escuchar es una forma de abrirnos nosotros mismos al otro y de recibir a este otro, su verdad y su mundo como diferente de nosotros, del nuestro.

Tal vez estén pensando que me estoy refiriendo a un nuevo tema para ser enseñado: hoy deberíamos enseñar a los estudiantes el respeto hacia otras culturas. Desde luego, esta es la cuestión. Pero, más generalmente, estoy hablando acerca del enseñar mismo. En una educación tradicional, el maestro expone la verdad y el alumno escucha su discurso. Si estamos de acuerdo con el hecho de que la verdad no es ni única ni universal, el maestro también debe escuchar la verdad del alumno. Claro, no estoy refiriéndome aquí a una mera verdad psicológica; por ejemplo, a algún problema que podría impedir al alumno escuchar y aprender la verdad ideal que yo, como maestra, debo enseñarle. Mejor dicho, estoy hablando de una verdad general que es propia de cada uno, y que tenemos que oír unos de otros.

El maestro y el alumno deben escucharse mutuamente para escuchar la verdad humana que cada uno expresa. Esto preserva la singularidad de cada uno, los dos estando en relación, y la relación horizontal entre sus subjetividades.

Mantener que sean dos en la relación entre maestro y alumno también permite que una comunidad de estudiantes se forme respetando la singularidad de cada uno, en lugar de constituir un grupo de álguienes compuesto por su sumisión a un supuesto tercero común ya existente, o a la autoridad del maestro funcionando como un tercer término.

Esto también nos permite mantener abierta la dimensión del presente y la presencia en la enseñanza. Desde luego, la experiencia pasada del maestro debe ser respetada, pero debe ser puesta al servicio de la relación 
presente entre maestro y estudiante, lo que no puede ser lo mismo que someter el tiempo presente y la entrada en presencia de los dos, al pasado.

Tal gesto conduce al/la maestro/a a cuestionar su propio pensamiento y a mantenerlo vivo y afectuoso. Podría aquí tomar prestada una palabra de Nietzsche afirmando que tal gesto puede proteger la enseñanza, y en primer lugar a cada maestro, del resentimiento y la venganza. De hecho, el resentimiento y la venganza adoptan la forma de confinar a uno mismo al pasado y de imponer al otro ese pasado. Nuestra manera de enseñar habitualmente implica un riesgo de resentimiento y venganza, que resulta de nuestra sumisión pasada a una educación autoritaria. Una vez más, no me refiero a una mera autoridad psicológica sino a la imposición de una cierta verdad como si fuera la verdad única y universal que debe aprenderse. Por supuesto, nuestra manera de someter a los estudiantes a nuestra propia educación pasada podría resultar también del hecho de que esta educación nos ha transformado en loros o máquinas, debido a la falta de escuchar a nuestro propio ser global, y por lo tanto nos tornamos incapaces de ser y de actuar mejor. Pero yo pienso que de todas formas existe resentimiento y venganza en nuestra manera de enseñar, que es un rechazo a abrirnos, a recibir la juventud de otros, especialmente de nuestros estudiantes, y así ayudarlos a hacer su propio camino hacia el futuro. Con demasiada frecuencia sometemos a los estudiantes a un tiempo pasado cuando enseñamos en lugar de despertarlos a su propio tiempo presente y a su tarea de prepararse para un futuro apropiado a cada uno, en ocasiones para un futuro compartido con el maestro como complemento. El maestro debe ser aquel que despierte a las personas más jóvenes al presente y al futuro, y no el que reprima y a veces mate, en nombre de la educación, los deseos de los estudiantes hacia el presente y el futuro, y sus presentimientos positivos y planes.

El maestro debe evitar tanto la imposición de una educación pasada como un nihilismo demagógico, lo que permitirá a los estudiantes pensar, decir y hacer cualquier cosa y todo, sin realizar la tarea de enseñar, esto es, de 
guiar, de ayudar al/la otro/a a descubrir su propio camino, a ingresar en el espacio y tiempo de su propia vida y de realizarla como un ser humano.

Enseñar no puede equivaler a imponer en el otro nuestro conocimiento, nuestra capacidad, incluso a través de una generosidad paternalista o maternalista. Enseñar no sólo consiste en hablar, sino también en ser capaces de permanecer en silencio, retirarse para permitir que el otro sea, se convierta y descubra su propio camino, su propio lenguaje.

Escuchar a quienes nosotros estamos enseñando también significa educarlos acerca del hecho de que la verdad de cada uno atraviesa el camino del otro, y que el asunto entonces consiste tanto en una apertura al/la otro/a, escuchar su verdad, como en ser fiel al propio camino, a la propia verdad. Es decir, ser capaces de retornar a nosotros mismos sin intentar convertirnos en el otro o pedirle al otro que se convierta en nosotros. El problema es entonces, en cada cruce, encontrarse con el otro como otro mientras sigan siendo dos.

De alguna manera, lo mismo puede decirse acerca del mundo. Tenemos que encontrarnos con el mundo, particularmente el mundo natural, como un otro, respetarlo en lugar de apropiarnos de él al interior de un solo mundo: nuestro mundo. La educación debería enseñar cómo respetar y contemplar al mundo, y no sólo cómo tomarlo y dominarlo, como ha sido muy frecuentemente el caso en nuestra cultura occidental.

\section{Pensar}

Luego de escuchar al otro y al mundo -y no sólo al mundo construido por nosotros- debemos volver a casa, volver a nosotros mismos, dentro de nosotros mismos. Enseñar nos exige ser, y no sólo tener conocimientos. Si la cuestión fuese sólo una cuestión de comunicar conocimiento, una máquina podría sustituir al profesor. Pero una máquina no puede reemplazar un cierto modo de ser, que el maestro ha conseguido a través de su propia capacitación, su propia experiencia de vida, su propia trayectoria. 
De hecho, lo más importante que un maestro tiene que transmitir es una manera de ser más que una manera de tener, una manera de ser alguien y no algo. Los maestros orientales me enseñaron que transformarme es la tarea más importante. Para lograr esta tarea, debemos tornarnos capaces de ser, de habitar, y no sólo de acumular conocimientos y técnicas. Los conocimientos y las técnicas que no funcionan hacia la transformación de nuestro ser amenazan con dañarnos a nosotros y al/los otro/s, en particular a nuestros estudiantes. Ellos contribuyen con un exilio de nosotros mismos, que transmitimos al/los otro/s en lugar de estar al servicio de convertirnos en nosotros mismos, de realizarnos.

Lo que es útil para esta tarea es pensar. Pensar no es una actividad lujosa reservada para unas pocas personas; pensar tiene que ser una tarea cotidiana para cada uno. Pensar es el momento de regresar a uno mismo. Pensar es el momento de construir el propio hogar, para habitar en nosotros mismos, para morar dentro del yo.

Tal morada debe unir en cada uno el pasado, el presente y el futuro. Tal morada puede existir sólo si nos dedicamos a construirla en cada momento. Nunca se construye de una vez y para siempre. Y es cuando la estamos construyendo que podemos permanecer en nosotros mismos. Así, la morada es propia de cada uno. $\mathrm{Y}$ es porque habitamos en nosotros mismos que podemos dialogar con el otro, con otros.

Pensar no es una mera tarea mental, ni un mero proceso técnico. Pensar es, o debería ser, una actividad de todo el ser. Con el fin de construir un hogar donde vivir, tenemos que usar nuestro cuerpo, nuestra imaginación e inteligencia. Habitar se refiere a todo lo que somos. El pensamiento puede coordinar, reunir, un pensamiento que no es sólo abstracto, lógico y técnico, sino más bien un pensamiento vivo que el corazón inspira y guía.

Pensar tiene que asegurar la vuelta a casa, el habitar dentro de uno mismo para descansar, para un devenir en uno mismo, para preparar las relaciones futuras con el otro, con el mundo. 
Pensar debe ser la tarea de todos. Pensar es especialmente la tarea del maestro. El/la maestro/a tiene que construir su propia morada con el fin de ayudar a otros a construir sus propias moradas. El maestro debe enseñar a los estudiantes cómo vivir y cómo encontrar y conservar una forma de pensar que le permita a cada uno, pero también a la humanidad presente y futura, vivir.

Esto implica que cuestionamos lo que muy a menudo consideramos como educación. Enseñar generalmente es entendido como transmitir -por no decir inculcar- a los estudiantes conocimientos, habilidades, técnicas. Entonces la enseñanza ya no tiene que ver con la humanidad como tal, y el maestro hoy en día se torna inútil, como es el caso para otros trabajadores. Una máquina puede convertirse en sustituto para él o ella. El maestro sigue siendo necesario sólo en tanto a lo que respecta a las relaciones entre humanos en nombre del pensamiento.

Unos cuantos riesgos ponen en peligro al pensamiento en nuestro tiempo. El imperialismo de las ciencias y técnicas, pero también el de las costumbres y hábitos, y el de opiniones o creencias. Y también una crítica arrogante que lleva al peor nihilismo y en ocasiones equivale a problemas psíquicos personales o a la decadencia cultural. Y un deseo de actuar meramente por actuar. Podría añadir algunos otros peligros que resultan de nuestra tradición occidental: otorgar primacía a la mente y olvidar la experiencia concreta y sensata; privilegiar la apariencia y la visibilidad en detrimento de la realidad invisible; querer dominar activamente sin aceptar recibir pasivamente, etc.

Así, uno de los peligros de nuestros tiempos es actuar meramente por actuar. Atreverse a actuar antes de pensar apunta, en parte, a superar nuestra lógica occidental y nuestras reglas. Enseñar entonces equivale a enseñar a actuar. Por lo tanto, uno podría decir que las relaciones entre el maestro y el estudiante no son realmente diferentes a las relaciones entre máquinas. Maestro y estudiante podrían reemplazarse por robots, y enseñar 
a través de un programa de software que se introduzca al interior del robot aprendiz.

Sin duda, actuar meramente por actuar representa uno de los peligros actuales para el pensamiento, y más en general para la humanidad, a través de competencias inútiles con máquinas. Actuar meramente por actuar es también un eslogan de los regímenes totalitarios que evitan el pensamiento e impiden las relaciones entre las personas a través del pensamiento. Actuar por actuar desarrolla una cultura tanto de la competencia como de la uniformidad. Además, algunas personas consideran al actuar por actuar como creatividad, mientras que la mayoría de las veces sólo equivale a lo que Freud llamó un paso al acto; uno también podría decir, la realización del ingreso inconsciente de un programa de software.

Ingresar a un programa de software hoy en día puede corresponder a una suerte de programa universal que sería apropiado para compartir entre todos nosotros. De esta manera, lo que en el pasado era pensar se convierte en un enfoque doctrinario con un único camino que, además, intenta brindarnos lo real mismo. De hecho, en nuestros tiempos, una manera de pensar podría sernos impuesta por medio de técnicas -entre otras cosas, la técnica de los medios de comunicación- que triunfan creando una opinión o creencia global que nuestra época necesitaría para su supervivencia. El privilegio exclusivo de las ciencias puede contribuir con esto, sobre todo ya que ahora se sostienen por métodos e instrumentos técnicos que desafian la experiencia humana. Las ciencias, las técnicas y la globalización, entre otras cosas, están todas reunidas para preparar un pensamiento global, la imposición de un único pensamiento: una suerte de totalitarismo sin representación ni representante, pero el cual es capaz de abolir la humanidad como tal. Cualquier pensamiento, cualquier manera de pensar puede obedecer a un enfoque único o doctrinario. El pensamiento nunca se perfila de una vez y para siempre. Y un único ímpetu o impulso nunca puede ser suficiente para que su programa pueda desarrollarse correctamente. 
Pensar siempre tiene lugar entre distintos caminos. Lo cual no quiere decir que se propague entre este y aquel: un campo $u$ otro, un pensador $u$ otro, alguna cultura u otra. Pensar requiere que uno permanezca fiel a su propio camino. Pero este camino requiere de un cuestionamiento constante, y cuestionar abre diferentes maneras posibles. El problema consiste en qué camino debe tomarse para permanecer fiel a su propio camino.

Pensar exige fidelidad y apertura, perseverancia e iniciativa. Pensar es un viaje, un viaje interior, que debe tener en cuenta el pasado y el futuro, y trazar un camino entre los dos. En el presente, el pensamiento cuestiona; notablemente hace preguntas sobre el camino. Una de las modalidades, uno de los métodos del cuestionamiento es abrirnos al otro. En nuestra tradición, es usual considerar el diálogo como una relación entre una persona que ya es capaz de pensar y otra persona que está aprendiendo cómo pensar, es decir, entre un maestro y un discípulo o estudiante que deben estar de acuerdo en una y la misma verdad, válida para los dos, y más allá de ellos. La verdad es entonces considerada como una objetividad externa a los dos, que no tiene nada que ver con su relación y sobre la cual ellos deben llegar a un acuerdo después de la discusión.

En mi opinión, un diálogo debe sostenerse entre dos sujetos diferentes que habitan en diferentes mundos y no comparten la misma verdad. En otras palabras, el diálogo no solamente ha de sostenerse entre dos sujetos más o menos competentes que deben llegar a un acuerdo acerca de un tercer término, un objeto. Un diálogo debe sostenerse entre un 'yo' y un 'tú' que son diferentes y deben permanecer diferentes; entonces el intercambio entre ellos apunta a iluminar el camino de cada uno y a preparar una posible coexistencia entre dos mundos, dos culturas, dos verdades, dos lugares o espacios, dos tiempos.

Puesto que estamos acostumbrados a transformar todo y a todos en representaciones, inmediatamente queremos imaginar quién soy yo y quién eres tú. Pero de esta manera eliminamos la diferencia entre yo y tú, y no se produce el diálogo. Si yo puedo imaginar quién o qué es el otro, y lo 
transformo en representaciones, él o ella dejan de existir como otro. El otro es quien escapa a mi manera de imaginar, y más generalmente a la de la cultura occidental. El otro como otro es el que puede cuestionar esta cultura sin abolirla. El otro indica lo que hay más allá de nuestra cultura sin aniquilarla, particularmente a través de la crítica.

El otro es aquel que puede cuestionarnos acerca de nuestro camino. El otro atraviesa nuestro camino y nos compele a cuestionarnos nuestro viaje.

El otro es aquel que nos enseña aunque nosotros seamos su maestro. Por ejemplo, en tanto ellos pertenecen a otra generación, los estudiantes desafian al/la maestro/a a descubrir una verdad diferente de la suya propia. Y esto es especialmente correcto hoy en día. Debido al imperialismo de las ciencias, al poder de las técnicas, a la globalización, lo que tenemos que enseñar hoy ya no es lo que nosotros hemos aprendido en tiempos pasados. Los temas a enseñar y también los métodos de enseñanza ahora son diferentes. Ya no se trata de enseñar cómo alcanzar un horizonte cultural ya existente. Ya no se trata de enseñar un mero criticismo. En cambio, se trata de pensar lo que la humanidad podria ser como tal, y qué cultura podría ser adecuada para la humanidad. Lo que tenemos que aprender en nuestros tiempos es cómo gobernar el mundo, como dijo Nietzsche. Y esta tarea no equivale a ejercitarse con programas gigantes, sintesis universalmente válidas, integraciones de datos dentro de paquetes de software que podrian usarse en una escala global. Todo eso nos aleja continuamente de aquello que tenemos que aprender: qué o quién es esencialmente un ser humano.

En nuestros tiempos, consideramos libertad de ser al hecho de vivir el día a día, rápidamente, fuera de cualquier responsabilidad. Todo eso agota y destruye nuestra humanidad, la que luego tratamos de restaurar con bienestar social, atención médica, pasatiempos culturales y un complemento de moralidad. La educación debería escapar a la necesidad de ser restaurada. Debe mantener la totalidad del ser -y Ser- y la capacidad de asegurar nuestro propio devenir. 
Además: pensar no puede conformarse con ir a favor o en contra, una alternativa que ha gobernado nuestra historia occidental. Este juego de sujeción y rechazo no es suficiente para pensar y enseñar el pensamiento. Recae sobre nosotros transformar estos juegos a favor y en contra en otra lógica, una lógica de la coexistencia en la diferencia: una diferencia que no puede ser cuantitativa, que no puede reducirse a un más o menos, o a un a favor o en contra. Una diferencia que es cualitativa, irreductible, y que debe tomar en consideración al otro como tal y no sólo a las cosas de nuestro mundo, un estado al que el otro ha sido reducido frecuentemente.

Entonces, la relación cuantitativa entre cosas materiales o espirituales es cambiada y se convierte en una diferencia cualitativa entre personas. A través de esta mudanza de la diferencia de un lugar a otro, entramos en otra era del pensamiento. Esto requiere que renunciemos libremente a una lógica cuantitativa -basada en el mismo, en el igual-a, en la identidad-con, etc.para tener acceso a otra lógica.

\section{Enseñar}

La lógica occidental hoy en día es desafiada de diferentes maneras: tecnología, globalización, las ciencias, los resultados de los trabajos de los pensadores y de la cultura occidental, etc. Estos diversos elementos podrian privarnos de cualquier lógica, de cualquier moderación, de cualquier razón si no adoptamos rápidamente otra lógica. Detenerse en la crítica no puede ser suficiente porque esto no nos permite acceder a otra lógica.

Debemos abandonar cierta forma de ser moldeados por nuestra lógica pasada a fin de alcanzar otra forma de Ser. Tal pasaje nos demanda al mismo tiempo disciplina y renunciación. Debemos renunciar a ciertas perspectivas en relación con lo absoluto, con lo infinito, con la dominación del mundo y con la razón misma. Pero este gesto es necesario de aquí en adelante para asegurar un futuro para la humanidad. Sin tal gesto, la crítica de nuestros valores corre el riesgo de terminar en el peor nihilismo, en la 
destrucción de la humanidad en sí, incluso a través del intento de imponer en una escala global lo que subsiste de nuestros valores: una especie de economía imperialista y una habilidad que no son específicas para la humanidad en sí misma.

Desde luego, existen otras culturas que tal vez tengan mayores recursos para asegurar un futuro para la humanidad. Sería deseable que nuestras culturas occidentales aprendan algunas verdades de ellas, en lugar de buscar superarlas con el riesgo de ser superadas ellas mismas.

No nos queda alternativa más que inventar otra lógica. Incluso la lógica de predicados, en la que se basan el pensamiento y la verdad occidentales, ya no puede resolver los problemas a los que nos estamos enfrentando: la entrada de la mujer en el discurso, los intercambios con otras culturas, los resultados de la cultura occidental, es decir, la influencia de la tecnología, la destrucción de la naturaleza, el solipsismo, ciertamente el aniquilamiento, del sujeto mismo. Nosotros los occidentales no podemos imponer nuestra lógica a todas las personas, a todas las personas del mundo. En cambio, debemos escuchar a aquellos que hablan, piensan y actúan de acuerdo con una lógica distinta a la nuestra. Todos nuestros gestos de integración más o menos paternalistas o maternalistas no equivalen al sentido que tenemos que descubrir y elaborar para el presente y el futuro: un sentido que pudiera ser compartido por todos los hombres y mujeres de nuestro planeta. De hecho, lo más importante que tenemos que aprender y enseñar es: cómo comunicarnos en la diferencia sin destruir nuestros propios valores, sin destruir al otro o a nosotros mismos. Por lo tanto, la necesidad de construir un hogar, una subjetividad, un ser, al cual podamos regresar, y donde podamos descansar y restaurarnos con el fin de ser capaces de dejar nuestro hogar, de abrirnos, de abrir nuestro mundo a una dimensión global, cuidando cada vez la otredad del otro.

La consideración y el respeto por la otredad del otro nos presentan a otra relación con la trascendencia. Y este es un paso crucial para alcanzar otra lógica y entrar en el multiculturalismo. En nuestra cultura occidental, la 
trascendencia se define como una dimensión que está fuera de nosotros, más allá de nosotros, ya sea una cuestión de cosas o del otro. La trascendencia se corresponde así con un absoluto, un ideal, un modelo más allá de nuestro alcance y que nos impone la ley desde arriba, fuera de nuestra capacidad de estimar y de experimentar su valor real. La mayoría de las veces en nuestra tradición, el/la maestro/a basa su autoridad en la relación que tiene con tal trascendencia, y su autoridad entonces se vuelve tan inalcanzable como la trascendencia misma. Desde la caída de los ídolos, este tipo de autoridad ya no funciona más. Pero tanto la caída de los ídolos del pasado como la falta de autoridad del maestro pueden llevarnos a situaciones peores. Pienso, por ejemplo, en el ejercicio de un poder carente de todo contenido por parte del maestro pero también por parte del alumno, o incluso por parte de la declaración de nuestras obligaciones por un grupo político, religioso o académico, que confunde una tarea administrativa para establecer autoridad sobre otros en nombre de la verdad. Todo esto es bastante aterrador aunque hoy en día se encuentre de moda. Y no es mera coincidencia que sea ahora una cuestión tan frecuente de totalitarismo. En efecto, estamos amenazados por todo tipo de totalitarismos más sutiles, imperceptibles y destructivos que aquellos que ya conocemos. Incluso estamos en peligro por la desesperación sobre un posible futuro para la humanidad. Ahora visualizar y construir nuestro futuro es el primer y más básico gesto hacia la trascendencia. Un gesto que permanece dentro de nuestro alcance y nos exige coraje y compromiso, pero no una fe ciega en un ideal inalcanzable o absoluto.

¿Cómo podríamos entonces reemplazar al ideal anterior de trascendencia y a la concepción de autoridad relacionada con ella? ¿Cómo podríamos liberar a la trascendencia de la prisión de entidades en la que ha sido fijada por nuestra cultura pasada? Desde ídolos espirituales en los que ya no confiamos y quienes, además, no son los mismos para todas las tradiciones y no favorecen la coexistencia y la paz entre los seres humanos. 
Reconocer y respetar al otro como otro puede corresponderse tanto a una trascendencia en el trabajo de la construcción de un futuro a nuestra escala como a la trascendencia que existe en alguien o en algo que permanece irreductible a nosotros sin estar, por todo eso, más allá de nuestro alcance, como es el absoluto del ideal o de Dios. El otro como otro está, de alguna manera, más allá de nuestro alcance que el mismo Dios puesto que aún Dios ha sido imaginado a partir de nosotros mismos. Dios está hecho a nuestra imagen. Dios es un sujeto, nuestra subjetividad, pero tan perfecto que no es posible ninguna comparación entre nosotros.

De alguna manera, podemos imaginar a Dios pero no podemos imaginar al otro como otro. Y si el negativo funciona sobre los atributos o los predicados de Dios en comparación con nosotros mismos, el negativo también funciona sobre el sujeto mismo cuando se trata de una cuestión de la otredad del otro, por ejemplo, la otredad que tiene lugar entre las identidades sexuales.

Podríamos decir que Dios es nuestra subjetividad en su estado puro o absoluto, mientras que no es el caso para el otro como tal: el otro es un otro, es decir, un sujeto diferente. Con el otro reconocido como otro, entramos en otra relación con la trascendencia. Una trascendencia cuya elaboración y salvaguarda ahora nos incumben, tal como ha correspondido a los filósofos griegos definir el tipo de trascendencia al que nosotros ahora tenemos que renunciar, que abandonar.

Entrar en otra relación con la trascendencia nos exige responsabilidad y esfuerzo. Ya no es una cuestión de acercarse a una perfección inalcanzable, situada fuera de nuestra subjetividad, de nuestro mundo, la cual sin embargo representa un modelo. La tarea más bien consiste en transformarnos en todo momento con el fin de respetar y cuidar la subsistencia y el devenir tanto de mí mismo/a como del otro, es decir, de dos sujetos radicalmente diferentes. El viaje es ahora más interno y el otro ya no es más aquel -el Uno- en quien yo deba convertirme, incluso cuando sé que este Otro es inalcanzable. Por el contrario, el otro es aquel a quien 
debo mantener diferente a mí. Es manteniendo la diferencia entre nuestras dos subjetividades que construyo trascendencia, la mía y, en la medida de lo posible, la del otro.

Tal elaboración no puede ser solamente, ni por encima de todo, mental como lo fue para la trascendencia pasada. Sucede a través de una transformación de todo nuestro ser. Esto implica la reunión de todo lo que somos: cuerpo, respiración, corazón, palabra, mente, y su transformación en una humanidad más perfecta a través de una transmutación, una transfiguración de la materia que, sin embargo, permanece materia. Una materia de otra naturaleza, menos sometida a la inercia, dotada de capacidades energéticas que se mantienen desconocidas cuando el cuerpo y la mente se mantienen separados y no entramos en una relación con el otro como otro. Lo que nos proporciona nuevos recursos energéticos.

Entonces puede existir la autoridad, una autoridad que resulta de convertirnos en humanos de un modo más completo. Una autoridad que no impone nada más que un cierto respeto, atención y cuestionamiento. Una autoridad que puede despertar al/los otro/s hacia un más allá con respeto a su estado presente y abrir un camino hacia el futuro. Una autoridad que invita al/la otro/a hacia una transformación de sí mismo o sí misma, a un devenir con vistas a la realización de la humanidad. Una autoridad que no equivale al ejercicio de un poder más o menos represivo, sino que se enseña a sí misma. Enseñar siempre tiene que ver con el devenir y con la trascendencia. La cuestión es cómo podemos enseñar un devenir hacia la trascendencia que no nos lleve otra vez a someternos a los ídolos pasados. En otras palabras: ¿cómo podemos enseñarle al/la otro/a a trascenderse a sí mismo o sí misma en lugar de someterse a una suerte de trascendencia ya existente?

Esta tarea me parece apropiada para nuestros tiempos. Nos permite coexistir en la diferencia. Nos impone un deber: hacer que la trascendencia exista más allá de la caída de los ídolos del pasado. Nos enseña cómo respetar la diferencia entre nosotros mismos, en lugar de que la diferencia 
se vuelva una fuente de conflictos y guerras, notablemente entre trascendencias o ídolos. Nos asegura un devenir de la humanidad a nuestra escala en una era en la que sentimos desesperación sobre la posibilidad para un futuro. Nos permite usar las ciencias, incluso las ciencias sociales y psicológicas, y también las técnicas de una manera que no resulte destructiva para la humanidad.

En los seminarios que sostuve en la Universidad de Nottingham, intenté enseñar de esta manera. Me gustaría agradecer a esta universidad una vez más, en mi nombre y en nombre de todos los participantes en los seminarios, por la hospitalidad ofrecida en esta ocasión.

Recibido: 21/04/2020

Revisado: 16/07/2020

Aprobado: 08/08/2020 